\title{
Laser resurfacing of skin flaps: an experimental comparison of three different lasers
}

\author{
${ }^{1}$ OMC Plastic Surgery, Rochester, MN \\ ${ }^{2}$ Division for Plastic Surgery \\ Department for Surgery \\ ${ }^{3}$ Department for Dermatology Mayo \\ Clinic, Rochester, MN
}

Srdan Babovic ${ }^{1}$, Uldis Bite ${ }^{2}$, Alina G. Bridges ${ }^{3}$, Ricky P. Clay ${ }^{2}$
Received: 8 December 2010

Accepted: 28 April 2011

Copyright (C) 2011 by Academy of Sciences and Arts of Bosnia and Herzegovina. E-mail for permission to publish: amabih@anubih.ba
Objective. The influence of Coherent Ultrapulse, TruPulse and Erbium: YAG laser skin resurfacing on survival of the skin flaps when performed simultaneously was evaluated. Material and methods. We used twelve female Yucatan minipigs in the study. Skin flaps including paniculus carnosus were raised on the animals' back. The flaps were sutured into the defect under tension. We designed 4 experimental groups: Control-Flaps only, Group 2-Flaps +4 immediate TruPulse laser passes, Group 3-Flaps +2 immediate Coherent UltraPulse laser passes, Group 4-Flaps - immediate $50 \mathrm{~J} / \mathrm{cm}^{2}$ total fluence with Erbium: YAG laser. Results. Flap survival in Control group was $98.8 \%$. There was no flap in Group 2 with complete survival. Survival of the flaps in Group 2 (Tru-Pulse) ranged from 75-90\%, with average flap survival area of $85.2 \%$. In Group 3 (UltraPulse) all 24 flaps had some area of necrosis. Flap survival in Group 3 ranged from $75-95 \%$, with an average of $85.6 \%$. In Group 4 (Erbium: YAG) flap survival area ranged from $70-95 \%$, with all 24 flaps with some area of necrosis, with average flap survival area of $87.3 \%$. There is a significant statistical difference in flap survival area between groups 2,3 and 4 versus Control $(\mathrm{p}<0.001)$. Conclusion. The results of our study suggest that laser resurfacing of skin flaps sutured under tension in the same operative session is detrimental for skin flap survival. We also found no significant difference in flap survival area between TruPulse, Coherent UltraPulse and Erbium: YAG laser treated flaps.

Key words: Laser resurfacing, Flaps, Ischemia injury.

\section{Introduction}

Rejuvenation of the face is an ever-changing area of aesthetic surgery. New technologies, new concepts and approaches are being introduced constantly. The pathophysiology of the changes induced in tissues is still being explored. The combination of different techniques in one procedure raises the additional question about the effects they have on tissues when applied concomitantly.

Simultaneous use of chemical peel and deep plane face-lift has been studied and no complications related to 
their combination have been reported (1). Reports about concomitant use of lasers and different esthetic procedures are available (2, $3,4,5,6)$, which show no additional complications arising from the combination of procedures. Our experimental data suggest that simultaneous use of pulsed $\mathrm{CO}_{2}$ laser resurfacing on skin flaps sutured under tension is detrimental for flap survival (7). Although the perfect method for treating photoaged skin remains elusive, the search for the best possible result, while minimizing complications, is a constant goal.

This study was designed to evaluate in a systematic and prospective manner the effects of Coherent UltraPulse, TruPulse and Erbium: YAG laser resurfacing on skin flaps sutured under tension when performed simultaneously.

\section{Material and methods}

Experimental animals

Female Yucatan minipigs were used in this study. This experimental animal is chosen because of histologic similarities with human skin $(1,8)$. We used twelve animals 3 months of age and $10-15 \mathrm{~kg}$ in weight.

\section{Experimental flaps}

Skin flaps, including paniculus carnosus (the flaps simulate deep plane facelift skin flaps), were raised on each animal's back. The flaps were oriented with the base toward the animal's spine. Flap size was $2 \times 6 \mathrm{~cm}$ (1:3). Eight flaps were elevated per animal and the treat- ment modality was randomly assigned for each flap.

The flaps were sutured under tension in the experimental protocol in order to mimic clinical practice. During face lift operative procedures the skin flaps are always sutured under tension and, in our model, flap tension was applied in a systematic fashion by suturing a smaller flap of $2 \times 4.5 \mathrm{~cm}$ in dimension into a defect measuring $2 \times 6 \mathrm{~cm}$. We designed four experimental groups: Control group, Group 2 (Tru Pulse Laser resurfaced), Group 3 (Coherent UltraPulse Laser resurfaced), and Group 4 (Erbium: Yag Laser resurfaced) (Table 1).

\section{Experimental protocol}

Skin flaps with paniculus carnosus were elevated on the animal's back as described above. The usual aseptic technique was followed - animal skin preparation with Betadine solution and the use of sterile instruments. One preoperative dose of Ancef 50 $\mathrm{mg} / \mathrm{kg}$ IM was administered to the animals. No flaps were elevated over the pelvic or scapular regions. Hemostasis was obtained with electrocautery and the flaps sutured in place with a two-layered 3/0 Vicryl closure. The distal $1.5 \mathrm{~cm}$ of each flap was resected and then sutured into the original flap defect under tension to simulate the tension placed on rhytidectomy flaps. Flap elevation and laser resurfacing was performed with the animals under general anesthesia.

The laser groups had flaps elevated and sutured in defects in same manner as the

Table 1 Experimental groups

\begin{tabular}{lll}
\hline Experimental groups & Flaps & Number of flaps \\
\hline Control & Flaps $2 \times 4.5 \mathrm{~cm}$ in $2 \times 6 \mathrm{~cm}$ defect & 24 \\
Tru Pulse Laser resurfaced & Flaps $2 \times 4.5 \mathrm{~cm}+$ immediate 4 laser passes & 24 \\
Coherent UltraPulse Laser resurfaced & Flaps $2 \times 4.5 \mathrm{~cm}+$ immediate 2 laser passes & 24 \\
Erbium: Yag Laser resurfaced & Flaps $2 \times 4.5 \mathrm{~cm}+$ immediate $50 \mathrm{~J} / \mathrm{cm}^{2}$ fluences laser pass & 24 \\
\hline Total & & 96 \\
\hline
\end{tabular}


control group. Immediately after insetting, the flaps were treated with the different lasers according to a randomization scheme of group assignment (Table 2).

Table 2 Randomization scheme

\begin{tabular}{|c|c|c|c|c|c|}
\hline \multirow{2}{*}{ Pig } & \multirow{2}{*}{ Side } & \multicolumn{4}{|c|}{ Position front to back } \\
\hline & & 1 & 2 & 3 & 4 \\
\hline 1 & Left & 3 & 2 & 4 & 1 \\
\hline 1 & Right & 1 & 4 & 2 & 3 \\
\hline 2 & Left & 4 & 1 & 3 & 2 \\
\hline 2 & Right & 2 & 3 & 1 & 4 \\
\hline 3 & Left & 1 & 3 & 2 & 4 \\
\hline 3 & Right & 4 & 2 & 3 & 1 \\
\hline 4 & Left & 2 & 4 & 1 & 3 \\
\hline 4 & Right & 3 & 1 & 4 & 2 \\
\hline 5 & Left & 3 & 1 & 4 & 2 \\
\hline 5 & Right & 2 & 4 & 1 & 3 \\
\hline 6 & Left & 1 & 4 & 2 & 3 \\
\hline 6 & Right & 3 & 2 & 4 & 1 \\
\hline 7 & Left & 2 & 3 & 1 & 4 \\
\hline 7 & Right & 4 & 1 & 3 & 2 \\
\hline 8 & Left & 4 & 2 & 3 & 1 \\
\hline 8 & Right & 1 & 3 & 2 & 4 \\
\hline 9 & Left & 2 & 3 & 4 & 1 \\
\hline 9 & Right & 1 & 4 & 3 & 2 \\
\hline 10 & Left & 4 & 1 & 2 & 3 \\
\hline 10 & Right & 3 & 2 & 1 & 4 \\
\hline 11 & Left & 3 & 4 & 1 & 2 \\
\hline 11 & Right & 2 & 1 & 4 & 3 \\
\hline 12 & Left & 1 & 2 & 3 & 4 \\
\hline 12 & Right & 4 & 3 & 2 & 1 \\
\hline
\end{tabular}

$1=$ control group, $2=$ TruPulse Laser group,

$3=$ Coherent UltraPulse group, 4=Erbium:YAG Laser group.

\section{Laser treatment}

Laser skin treatment was delivered using two different operative $\mathrm{CO}_{2}$ and Erbium: YAG lasers. In Group 2 the Tru-Pulse Laser System with a 4-mm hand probe was used. Four laser passes were performed with 500 $\mathrm{mJ} / \mathrm{cm}^{2}$ of energy to the skin flaps in every pass. In Group 3 we used a Coherent UltraPulse with a CPG scanner. We delivered a fluence of $300 \mathrm{~mJ} / \mathrm{cm}^{2}$ with density of 5 in two passes to create a similar depth of injury. In Group 4 we used an Erbium: YAG laser at $2940 \mathrm{~nm}$ wavelength. We delivered total fluences of $50 \mathrm{~J} / \mathrm{cm}^{2}$ in 3 passes of $17 \mathrm{~J} / \mathrm{cm}^{2}$ with $30-50 \%$ scanner pattern overlap.

The skin was treated with wet and dry sponges between laser passes. Care was taken to treat the entire surface of the flap uniformly. The settings for the different laser systems were chosen to correspond to the settings used in clinical facial skin laser resurfacing. We decided to use the most frequently used settings published in current laser literature.

Close daily monitoring was secured in the postoperative period and any sign of animal distress was properly treated. Wound cleaning with $0.9 \%$ Normal Saline and application of Bacitracin antibiotic ointment was provided daily in the first postoperative week.

\section{Observations}

Flaps were examined and photographed weekly and flap viability evaluated. Photographs were taken from the same distance every time. Skin flap necrosis area was defined postoperatively by development of full thickness necrotic eschar. An area of flap necrosis or survival was measured from the photos and presented as a percentage of the total flap surface area. Total observation time was 4 weeks after the procedure. Punch knife biopsies were taken from 2 flaps in each group immediately after resurfacing, one week post-resurfacing and at 4 week intervals and H\&E stains prepared for histology and evaluation of changes in flap skin thickness, capillary and collagen pattern. Histology evaluations were performed in a blind fashion by the same dermatopathologist (AGB). 


\section{Statistical analysis}

The experiment was carried out on twelve experimental animals in order to examine the difference between four different treatments. As each pig received multiple treatments, we assigned the four treatments in such a way that we systematically checked for variations in necrosis due to the characteristics of the pig, characteristics of the side (right or left) of the pig, and characteristics of the position of the flap on the pig. We used three separate pairs of Latin squares. Each pair of Latin squares consisted of four pigs. One of the squares corresponded to the right hand side of the pig, and the other to the left hand side. Each of the three pairs of Latin squares had a different order of treatment assignments.

\section{Results}

Flap survival in flaps in the Control Group was $98.8 \%$. Only one flap developed distal $5 \%$ necrosis. There was no flap in Group 2 with complete survival. Survival of the flaps in Group 2 (Tru-Pulse) ranged from $75-90 \%$, with average flap survival area of $85.2 \%$. In Group 3 (UltraPulse) all 24 flaps had some area of necrosis. Flap survival in Group 3 ranged from $75-95 \%$, with an average of 85.6\%. In Group 4 (Erbium: YAG) flap survival area ranged from $70-95 \%$, with all 24 flaps with some area of necrosis. Mean survival area was $87.3 \%$. There is a significant statistical difference in flap survival area between Groups 2,3 and 4 versus the Control $(\mathrm{p}<0.001)$. There is no statistically significant difference between laser groups: TruPulse vs. UltraPulse $\mathrm{p}=0.84$, TruPulse vs. Erbium: YAG $\mathrm{p}=0.31$ and UltraPulse vs. Erbium: YAG p $=0.42$.

\section{Histology \\ Immediately after flap elevation and laser treatment}

Control: Stratum corneum and epidermis are unremarkable. There is a mild superfi- cial and deep perivascular and interstitial lymphohistiocytic infiltrate. The adnexal structures are unremarkable. Full thickness epidermal necrosis was found in histological specimens in all three laser groups. There is a mild superficial and deep perivascular and interstitial lymphohistiocytic infiltrate. The adnexal structures are unremarkable. There was no visible histologic difference between Coherent Ultrapulse, Trupulse and Erbium: YAG immediate specimens.

\section{Seven days post flap elevation and laser resurfacing}

Control Group specimens obtained at this time point showed the same characteristics as originally described. Erbium: YAG group showed serum crust, reepithelialization of the epidermis. There is a mild superficial and deep perivascular and interstitial lymphohistiocytic infiltrate. The adnexal structures are unremarkable. Coherent Ultrapulse and Trupulse specimens presented same as Erbium: YAG group at 7 days.

\section{One month after flaps and laser resurfacing}

Control Group specimens obtained at this time point showed the same characteristics as originally described. TruPulse specimens had mild basketweave orthohyperkeratosis, mild irregular acanthosis, mild hyperpigmentation of the basal layer, and melanophages in the papillary dermis, fibrosis and proliferation of blood vessels in the papillary dermis. There is a mild superficial and deep perivascular and interstitial lymphohistiocytic infiltrate. The adnexal structures are unremarkable. CoherentUltrapulse and Erbium: YAG presented the same as at 4 weeks. TruPulse specimens.

\section{Discussion}

Introduction of laser technology in laser rejuvenation has opened new horizons, set 
new goals, and defined new perspectives. Also, there is so much to learn about the effects that laser energy induces in tissues. Continuous research efforts constantly improve our knowledge of laser effects on tissue.

This study was designed on a well established experimental model used in prior skin resurfacing experiments $(1,2,7,8)$. The vascularity of the axial pattern flaps in the Yucatan minipig is well established. Flaps of $6 \mathrm{~cm}$ length have been shown to survive even when a random pattern was used (9).

The depth of the dermal injury appears to be similar with a 35\% TCA peel and the Ultra-Pulse laser (8). The pathophysiology of laser resurfacing producing thermal damage is likely to be responsible for such a difference in flap perfusion and the development of skin necrosis. Histologic data from our study proved the depth of the thermal ablation to the level of the papillary dermis, consistent with published data on the same experimental model $(3,8)$. Reepithelialization time and histologic sections one month post laser resurfacing in our study were also concordant with previously published data $(1,3,7,8)$.

Despite the results from several studies indicating no additional complications when simultaneous face-lift and laser resurfacing have been used, multiple experimental reports suggest a different approach $(2,3$, $7,10)$. Our previous experiment suggested that laser thermal injury is detrimental for flap survival when the flap is sutured under tension. Our current data suggest that the specifics of different laser energy do not significantly change that influence.

Different laser pass settings and different numbers of passes have different effects on skin. However, the cumulative effect is not a simple sum of the energy delivered. More passes at a lower setting appear to have deeper penetration in the skin (11). It is yet to be determined what minimal threshold of energy is needed to induce irreversible perfusion changes in skin flaps (12).
Histologic changes in the skin after laser resurfacing continue to occur beyond the initial changes. Most likely, neocollagen formation and shrinkage can be significant factors in obtaining long lasting results of $\mathrm{CO}_{2}$ laser resurfacing $(11,13,14)$. Also, skin contraction after laser resurfacing correlates directly to the zone of dermal coagulation induced. That correlation remained in both pulsed $\mathrm{CO}_{2}$ and Erbium: YAG laser resurfacing (9). Similar observations were made in our study regarding the resurfaced flap perfusion, the corresponding energies of three different lasers induced comparable amounts of skin flap necrosis. There is a possibility that pretreatment of skin flaps with retinoic acid could improve flap survival. Four to six weeks pretreatment of skin with retinoic acid before laser resurfacing decreased the depth of injury induced by laser resurfacing, and the wounds seemed to heal faster (14).

There are multiple factors influencing postoperative outcome in laser skin resurfacing $(16,17)$. Promising results have been obtained with Erbium: YAG laser resurfacing, as well as with a combination of erbium and carbon dioxide lasers $(18,19)$. In our experiment (erbium resurfacing of skin flaps sutured under tension induced the least amount of necrosis, but there was no statistically significant difference when compared with two other experimental groups. One cannot help asking: Would retinoic acid pretreatment and/or less skin flap tension be beneficial for skin flap survival? The ever-changing field of laser skin resurfacing amazingly brings new technology with decreased morbidity $(18,20)$.

\section{Conclusions}

The results of our study suggest that laser resurfacing of skin flaps sutured under tension in the same operative session is detrimental for skin flap survival. There was no signifi- 
cant difference in flap survival area between TruPulse, Coherent UltraPulse and Erbium: YAG laser treated flaps. Laser treatment induced histologic changes in skin flaps that were similar, corresponding to the similar energy delivered, regardless of laser type used.

Authors' contributions: Conception and design: SB and UB; Acquisition, analysis and interpretation of data: SB, AB and UB; Drafting the article: SB; Revising it critically for important intellectual content: $\mathrm{SB}$ and RPC

Conflict of interest: The authors declare that they have no conflict of interest. This study was not sponsored by any external organization.

\section{References}

1. Dingman DL, Hartog J, Siemionow M. Simultaneous Deep plane face lift and trichloracetic acid peel. Plast Reconstr Surg.1994;93:86-93.

2. Mayl N, Felder DS. $\mathrm{CO}_{2}$ laser resurfacing over facial flaps. Aesthet Surg J. 2004;17(5):285-92.

3. Guyuron B, Michelow B, Schmelzer R, Thomas T, Ellison MA. Delayed healing of rhytidectomy flap resurfaced with $\mathrm{CO}_{2}$ laser. Plast Reconstr Surg. 1998;101:816-9.

4. Alster TS, Doshi SN, Hopping SB. Combination surgical Lifting with ablative laser skin resurfacing of facial skin: a retrospective analysis. Dermatol Surg. 2004;30(9):1191-5.

5. Jackson IT, Yavuzer R, Beal B. Simultaneous face lift and carbon dioxide laser resurfacing: a proven technique. Plast Reconstr Surg. 2001;108(3):802-3.

6. Achauer B.M., Adair S.R., VanderKam M. Combined rhytidectomy and full-face laser resurfacing. Plast Reconstr Surg. 2000;106(7):1608-11.

7. Babovic S, Clay RP, Battey MJ, Trevino MT, Bite $\mathrm{U}$ : The effect of laser resurfacing on flap survival. (Non published data).

8. Minoli JJ, Barton FE. A comparison of the histologic effects of chemabrasion, dermabrasion and laserabrasion in the minipig. Aesthetic Surgery J. 1998;18(1):11-8.

9. Stell, P. The pig as an experimental model for skin flap behavior: A reappraisal of previous studies. British J Plast Surg. 1977;30:1-8.

10. Adckok D, Paulsen S, Katzen T, Harper L, Nanney LB, Shack RB. Simultaneous cutaneous flap elevation and skin resurfacing in the rabbit model. Aesthetic Surg J. 1999;19(5):375-80.

11. Grover S, Apferberg DB, Smoller B. Effects of varying density patterns and passes on depth of penetration in facial skin utilizing thew carbon dioxide laser with automated scanner. Plast Reconstr Surg. 1999;104:2247-52.

12. Carniol PJ. The importance of blood supply in combination rhytidectomy and full-face carbon dioxide laser resurfacing. Arch Facial Plast Surg. 2002;4(4):227-33

13. Kirn DS, Vasckonez HC, Cibull ML, Fink BF. Skin contraction with pulsed $\mathrm{CO}_{2}$ and Erbium: YAG laser. Plast Reconstr Surg. 1999;104:2255-60.

14. Rosenberg GJ, Brito MA, Aportella R, Kapoor S. Long-term histologic effects of the $\mathrm{CO}_{2}$ laser. Plast Reconstr Surg. 1999;104:2239-44.

15. McDonald WS, Beasley D, Jones C. Retinoic acid and $\mathrm{CO}_{2}$ laser resurfacing. Plast Reconstr Surg. 1999;104:2229-35.

16. Weinstein C, Ramirez OM, Pozner JN. Postoperative Care following $\mathrm{C}_{2}$ laser resurfacing: avoiding pitfalls. Plast Reconstr Surg. 1997;100(7):1855-66.

17. Bernstein LJ, Kauvar AN, Grossman MC, Geronemus RG. The short and long term side effects of carbon dioxide laser resurfacing. Dermatologic Surg. 1997;23(7):519-25.

18. Weinstein C. Erbium laser resurfacing: Current concepts. Plast Reconstr Surg. 1999;103:602-16.

19. Alister TC. Cutaneous resurfacing with $\mathrm{CO}_{2}$ and Erbium: YAG lasers: Preoperative, intraoperative and postoperative considerations. Plast Reconstr Surg. 1999;103:619-32.

20. Schwartz RJ, Burns AJ, Rohrich RJ, Barton FE, Byrd HS. Long-term assessment of $\mathrm{CO}_{2}$ facial laser resurfacing: Aesthetic results and complications. Plast Reconstr Surg. 1999;103(2):592-601. 